\title{
Effects of 'in-service' conditions - mimicked hull roughness ranges and biofilms- on the surface and hydrodynamic characteristics of Foul-Release type coatings
}

\author{
I.A. Yeginbayeva ${ }^{\mathrm{a} *}$, M.Atlar ${ }^{\mathrm{b}}$, S.Turkmen ${ }^{\mathrm{c}}$, Haoliang Chen $^{\mathrm{d}}$ \\ a Department of Mechanics and Maritime Sciences (M2), Chalmers University of Technology, \\ Gothenburg, Sweden, SE-412 96 \\ ${ }^{\mathrm{b}}$ Department of Naval Architecture, Ocean and Marine Engineering, University of Strathclyde, \\ Glasgow, UK, G1 $1 X Q$
}

'School of Engineering, Marine, Offshore and Subsea Technology Group, Newcastle University, Newcastle, UK, NE1 7RU

${ }^{d}$ International Paint Singapore Pte Ltd, Singapore

*Corresponding author: Dr Irma Amangeldykyzy Yeginbayeva

Address: Department of Mechanics and Maritime Sciences (M2), Chalmers University of Technology, Lindholmen campus, Saga building, 4 Hörselgången street, Gothenburg, Sweden, SE-412 96

E-mail :yeginbayevai@gmail.com

Mobile: +47 47769670

Prof Mehmet Atlar

Address: Department of Naval Architecture, Ocean and Marine Engineering, University of Strathclyde, Henry Dyer Building, 100 Montrose Street, Glasgow, UK, G4 0ZL

E-mail: mehmet.atlar@strath.ac.uk

Telephone: +44 (0)1415484947

Dr Serkan Turkmen

Address: School of Engineering, Marine, Offshore and Subsea Technology Group, Newcastle University, Armstrong Building, Queen Victoria Road, Newcastle, UK, NE1 7RU

E-mail: Serkan.Turkmen@newcastle.ac.uk

Telephone: +44(0)1912085862

Dr. Haoliang Chen

Address: International Paint Singapore Pte Ltd, 21 Tuas South Street 3

E-mail: haoliang.chen@akzonobel.com

Telephone: +65 65948854 


\section{Effects of 'in-service' conditions - mimicked hull roughness ranges and biofilms- on the surface and hydrodynamic characteristics of Foul-Release type coatings}

To develop a better understanding of 'in-service' performance of modern marine coatings, the paper explores combined effects of different roughness ranges of foul-release coating (FRC) and light biofouling (slime) on the surface, boundary layer and drag characteristics under a range of 'in-service' conditions. Natural and laboratory biofilms were grown dynamically on FRC panels by exposing panels in facilities dedicated to realistic fouling culturing. The boundary layer experiments were conducted in a circulating water tunnel. Boundary layer similarity-law scaling is used to predict the combined effects of coating roughness and biofilms on the added frictional resistance $\left(\% \Delta C_{F}\right)$ and added required effective power $\left(\% \Delta P_{E}\right)$ for a benchmark KRISO container ship (KCS) and bulk carrier. The increase in $\% \Delta P_{E}$ due to the presence of biofilms on commercial FRC is estimated to be between $6.5 \%$ and $16 \%$ depending on the biofilm type, biofilm thickness and percentage coverage. Significant increases in effective power are estimated for non-fouling control primers with heavy fouling. Moreover, the paper suggests updated roughness allowances $\left(\Delta C_{F}\right)$ for two vessel types assuming FRCs on their hulls with more representative hull roughness ranges and fluffy biofilms.

Keywords: coatings ‘in-service’ condition, fouling, biofilms, skin friction drag, ship power penalties 


\section{Introduction}

Maintaining the efficiency of a ship is strongly linked with the requirement to utilize an effective fouling control solution to prevent biofouling accumulation. It is a well-known fact that there are more than 4000 marine species identified as biofouling and sessile organisms (Yebra et al. 2004). The indirect effects of fouling on climate change, which include, but are not limited to, an increase in hull frictional resistance and greenhouse gas (GHG) emissions encourge a need to apply preventive actions such as improving current coating technologies and introducing new coating solutions. Hence, there is a particular need for improved eco-friendly and high-performance coatings with wider target range of vessel types and operational profiles.

Fouling is a dynamic phenomenon, the degree of which changes with the time of the year, geographical location, water parameters, water depth, and flow characteristics. Unlike hard fouling, a complete definition of microfouling which is often referred to as biofilm/slime is rather complex. For ship owners, hydroelectric and underwater power generator sources, slime is recognized as a serious issue, causing penalties in increased frictional resistance and resulting in energy losses (Townsin 2003); (Andrewartha et al. 2010); (Walker et al. 2013). Both full-scale ship trials and laboratory studies have demonstrated the detrimental effect of biofilms on hydrodynamic drag (Murphy et al. 2019) and hence ship performance (Schultz and Swain 2000), (Lewthwaite et al. 1985). Early research into understanding the plant fouling and biofilm effect of friction started with McEntee (1915), followed by Benson et al. (1938), Denny (1951) and Watanabe et al. (1969). Lewkowicz and Das (1986) simulated the slime growth by attaching nylon tufts to a rough flat plate and measured the mean and turbulence data for this surface. Their results collected in zero-pressure gradient flow revealed that the slime alone increased the skin friction by an average of $18 \%$ when the results were compared to background rough flat plate data. Laboratory and full-scale ship trials conducted by Haslbeck and Bohlander (1992) showed that biofilm can significantly increase drag: a significant change in power consumption, ranging from 8 to 18\%, was measured by power trials before and after underwater cleaning to remove microbial biofilms from the hull. Schultz (2004) compared the frictional resistance of fouling release and biocidal hull coatings in unfouled, fouled, and cleaned conditions and found a significant increase in their skin friction coefficient $\left(C_{f}\right)$ for surfaces covered in slime. Later, these laboratory results were translated into full-scale ship resistance and powering predictions for an Oliver Hazard Perry class frigate (Schultz 2007). His estimates, which showed good agreement with the full-scale power trials, indicated that slime films can lead to significant increases in resistance and powering. The detailed study of Schultz et al. (2011), which enabled the projection of an economic impact of hull fouling over 15 years and over the whole DDG-51 class of ships, estimated an enormous effect of fouling on fuel consumption and subsequent operating costs eg \$1.2M per ship due to heavy slime.

It is believed that surface properties ranging from structure, morphology and material properties affect the development of biofilms from initial larval phases to the final coverage state. According to Characklis (1973) and Rodney (2002), the extent of microbial colonization increases with increasing roughness. This is attributed to diminished shear forces in the pockets of the rough surfaces, which provides a protective habitat to the living organisms. A number of reports in the literature also demonstrate that the strength of the attachment of diatomic biofilms increases with increasing hydrophobicity or low surface energy (Anderson et al. 2003), (Holland et al. 2004), which forms the basis for non-toxic fouling release coatings. Zargiel and Swain (2014) 
investigated the adhesion strength and settlement of diatomic cells on one ablative coating, four foul-release coatings (FRC) and one control surface (epoxy barrier coating) in static and dynamic conditions. Their results showed qualitative and quantitative differences between biofilms grown in static and dynamic conditions depending on the coating and sampling dates. In general, panels which developed biofilms under static conditions, had fewer and poorly attached diatoms

A considerable amount of literature has been published on fouling growth on coated surfaces either in static or dynamic immersion conditions, but mainly in rotary setups with tangential velocities (Lindholdt et al. 2015), (Schultz et al. 2015). Without question, these tools are useful approaches towards realistic exposure in terms of generating dynamic conditions. However, ideally, it is preferred to do it in-situ, with natural seawater and under ship navigation conditions, both regarding the speed and shear direction of the flow.

The added frictional resistance of biofilms is among the least understood of all fouling categories. This is mainly because of an ever-changing nature of biofilms with the time of the year, surrounding environment and characteristics of surface of contact or surface roughness. Based on this, it can be hypothesized that biofilms grown on the same coating types but under laboratory and field conditions will be different which might result in different drag performance. Therefore this study seeks to understand the drag associated with biofilms grown on non-biocidal FRCcoated panels under two exposure conditions. To the authors' best knowledge, most of the studies in the field are limited to growing biofilms statically or at relatively low shear velocities on coatings with relatively smooth finishes. In order to address this knowledge gap, cultivation of biofilms on FRC-coated surfaces was carried out by using two different dynamic biofilm growth methods: one being a natural exposure of coatings at sea; and other under laboratory conditions. A recent paper by Yeginbayeva I A and Atlar (2018) investigated the effect of mimicked 'hull' roughness ranges on the drag and roughness characteristics of panels coated with modern nonbiocidal FRC and biocidal antifouling coatings in their clean (ie, unexposed to biofilm) conditions. The current paper further expands and hence complements the above-mentioned study by investigating the combined effects of FRC coating roughness and biological roughness (biofilms) on the drag. This approach reveals how different exposure conditions affect the biofilm development, its coverage, and subsequent biofilm resiliency during drag tests. Moreover, the results of this study are used to predict the potential impact of 'in-service' coating conditions on the hydrodynamic performance of a KRISO container ship (KCS) and bulk carrier using a similarity law procedure given by Granville (1987). Given the different exposure environments, different seasons, microfouling intensity, which is subject to time, experiments at sea and lab conditions cannot be related directly. This study seeks to assess the hydrodynamic drag produced by biofilms and therefore provides no taxonomic identification.

\section{Materials and Methods}

\subsection{Test panel}

Test coatings were applied to flat test panels. These are also called standard 'UNEW (Newcastle university) test panels' with a base made of perspex. The drawing for the standard test panel with its main dimensions is given in Figure 1. As shown in the figure, the shaded area of the panel with a dimension of $218 \mathrm{~mm}$ x $598 \mathrm{~mm}$ was coated with selected coatings. The design and manufacture 
of facilities such as a dynamic slime farm, strut assembly and test bed of the Emerson Cavitation Tunnel used in the study are based on the dimensions of the panel.

Figure 1. Main scantlings for UNEW's standard test panel. All dimensions are in millimeters (mm).

\subsection{Dynamic biofilm growth methods}

Considering the limitations of previous studies, this study explored the ways of simulating biofilms on coated flat panels under more realistic flow conditions. As a result, two dynamic systems have been designed, built and used to cultivate biofilms on FRCs.

In the first system, standard test panels were coated with FRC and exposed to natural biofilms by attaching them to the specially designed and manufactured strut assembly. The strut arrangement was deployed from the moonpool plug of Newcastle University's research catamaran, 'Princess Royal' as shown in Figure 2a. At any given time, the strut arrangement can accommodate up to eight test panels (Figure $2 \mathrm{~b}$ ). The research catamaran operates around the port of Blyth, Northeast coast of England $\left(55.1182^{\circ} \mathrm{N}, 1.4960^{\circ} \mathrm{W}\right)$. Generally, the vessel is active for about $35 \%$ of the year in the marine coastal environment and has a typical operational speed between 6 to 20 knots. In addition to experiencing the dynamic conditions due to the movement of the ship, the test panels on the strut experience real sea conditions in terms of the depth of submergence, the availability of nutrients, salinity and sea temperature. The strut assembly also enables inspection of the biofilm development at any time as illustrated in Figure 2b. Detailed information on the design, manufacture and operation of the strut assembly can be found in (Atlar et al. 2015).

a)

Figure 2. A view of strut assembly to collect slime samples on test panels: a) Strut deployed when 'Princess Royal' is in a dry dock; b) Test panels under inspection

Growing biofilms under laboratory conditions, as in the second system, has the advantages of a controlled environment. For example, the ability to control salinity, temperature, light, nutrient levels, etc, allows experimental flexibility and accelerated growth rates. Therefore, the dynamic slime farm was specifically designed and manufactured to accommodate up to four standard test panels. The slime farm is a closed loop system consisting of the main testing section, pump and reservoir tank (see Figure 3). A removable internal flow separator made from perspex is placed longitudinally in the centre plane of the testing section and used: firstly, to accelerate the flow in the testing section; secondly, to provide a watertight chamber for an aquatic, 360-degree fluorescent tubes which are located horizontally and fixed in a desired position with the help of clamps. Apart from being the watertight chamber for the lighting, the aquatic box also serves as a lid to cover the working section of the tank to isolate the air and ensure the safe operation. The flow in the slime farm was ensured by circulating the seawater collected from the North Sea using a 3kW corrosion-resistant pump (ARBO 2016). Detailed information on the slime farm can be found in the lead author's PhD thesis (Yeginbayeva I.A 2017). 
Figure 3. The general arrangement of a laboratory-based slime farm

\subsection{Definition of test panels \& test matrix}

In total 10 coated panels including replicate panels were prepared for the experiments. These include: two primer coated panels, four FRC coated panels with 'normal' finish, two FRC coated panels with 'low' density of mimicked hull roughness, two FRC coated panels with 'high' density of mimicked hull roughness panels which were exposed to biofilm growth using the previously described two biofilm cultivating systems. FRC coated panels are amphiphilic coating system Intersleek ${ }^{\circledR} 900$, whereas the primer panels are Intershield ${ }^{\circledR} 300$.

Intersleek ${ }^{\circledR} 900$ (grey in colour) was applied in duplicate onto acrylic-based test panels by using standard procedures (airless spray). These panels were used to represent relatively smooth, laboratory prepared test pieces or 'normal' finish. These two replicate panels were exposed to biofilm growth under natural conditions using the research vessel's strut assembly. The panels are specified as 'I_900_Field1' and 'I_900_Field2' to refer to the natural biofilms on the first and second replicate of Intersleek ${ }^{\circledR} 900$, respectively.

Further, the same Intersleek ${ }^{\circledR} 900$ was applied in duplicate using standard procedures and exposed to the biofilm growth in the slime farm. These replicate panels are referred to as 'I_900_Lab1' and 'I_900_Lab2' to address the laboratory biofilms on FRC types with 'normal' finish. Average Hull Roughness (AHR) given in $R_{t} 50$ values (the maximum peak-to-trough height taken over 50 mm sample length) for FRC coated panels with 'normal' finish applied in the laboratory onto flat panels using a 'normal' application procedure were in the range of 40-50 $\mu \mathrm{m}$. The paint colour difference between the field and laboratory exposed Intersleek ${ }^{\circledR} 900$ panels that limits the strength of comparison across treatments was due to a material constraints.

Additionally, primer coatings were prepared in duplicates to act as control surfaces with no inherent fouling control properties and deployed in the slime farm alongside FRCs with 'normal' finish. The first and second replicate panels of primer coating with laboratory fouling samples are referred to as 'Primer_Lab1' and 'Primer_Lab2', respectively. The above-mentioned panels were part of the ' 1 st campaign' of laboratory experiments and were used as control surfaces.

Finally, Intersleek ${ }^{\circledR} 900$ (red in colour) was applied in duplicate to acrylic-base test panels to provide surfaces with roughness values that are more representative of the typical average hull roughness range observed on commercial ship hulls. To begin the application process to mimic hull roughness ranges of ships, artificial roughness in the form of sand grit of $25 \%$ and $50 \%$ by weight was introduced into the anticorrosive coats of panels coated with Intersleek ${ }^{\circledR} 900$ scheme. These two different sand grit levels were assumed to represent relatively 'low' and 'high' densities of hull roughness scenarios. A detailed description of an approach to mimic the levels of physical roughness experienced on ship hulls are given in the authors proceeding paper (Yeginbayeva I A and Atlar 2018). These panels were exposed in the slime farm. The first replicate of typical FRC type with 'low' and 'high' roughness densities are denoted as 'I_900_Lab_low1'and 'I_900_Lab_high1' respectively to address the combined effects of mimicked roughness densities and samples of laboratory biofilms. Whereas, the names for the second replicate of the same coating types come with coating identifiers: 'I_900_Lab_low2'and 'I_900_Lab_high2'. The above-mentioned panels were part of the ' $2{ }^{\text {nd }}$ campaign’ of laboratory experiments. Figure 4 shows 
photos of all test panels before and after dynamic exposure in the natural and laboratory habitats. FRC coated panels with 'low' and 'high' density of roughness exhibited $R_{t} 50$ values in the range of 197-240 $\mu \mathrm{m}$, which can be regarded as the typical average hull roughness range for commercial vessels based on the experience of International Paint Ltd and their analysis of a dataset of 845 individual hull roughness surveys carried out between 2003 and 2014 (Stenson et al. 2014).

\subsection{Biofilm growth and parametrization}

\section{Field grown biofilms at sea}

The deployment of panels on the strut assembly of 'Princess Royal' took place on the $17^{\text {th }}$ of September 2015. The biofilm was grown under natural dynamic conditions for around 6 months. The average Blyth water temperature varied from a minimum of $6^{\circ} \mathrm{C}$ in March to a maximum of $14^{\circ} \mathrm{C}$ in September. As can be seen from Figure 4a, Intersleek ${ }^{\circledR} 900$ duplicates (I_900_Field1, I_900_Field2) developed a noticeable amount of patchy biofilms with some areas being void. No macro fouling was observed.

\section{Laboratory-grown biofilms in the slime farm}

Fouling was grown in a channel flow in the slime farm. The channel gap height in the slime farm is $50 \mathrm{~mm}$ and a honeycomb ('Tubus-Bauer') is inserted so that velocity distribution upstream of the test panels is smoother. After the honeycomb, the hydrodynamic entry length of $60 \mathrm{~mm}$ was ensured to establish a fully developed flow just before the test panels. The slime growth was carried out at $30 \mathrm{~Hz}$ pump motor frequency. According to the flow measurements carried out by Dantec's portable LDV or 2D FlowExplorer, the streamwise velocity over the test panels was $\approx 0.5 \mathrm{~m} / \mathrm{s}$, which coresponds to a flowrate of $\mathrm{Q} \approx 30 \mathrm{~m}^{3} / \mathrm{h}$. At the beginning of each biofilm growth campaign, the slime farm was inoculated with the culture extracted from the hull of the research vessel to ensure similar biofouling species as on an active vessel. Two duplicate panels coated with Intersleek ${ }^{\circledR} 900$ (grey in colour) and primer coating were exposed to the circulating seawater in the slime farm. The $1^{\text {st }}$ campaign of the slime growth tests started in February 2016 and ran for around one month. Guillard's (F/2) marine water enrichment solution without silica was added during the slime growth to stimulate a quick growth rate. During the whole biofilm growth period, the temperature in the slime farm was between $19^{\circ} \mathrm{C}-21.5^{\circ} \mathrm{C}$. Photos in Figure $4 \mathrm{~b}$ and $4 \mathrm{c}$ show duplicates of Intersleek ${ }^{\circledR} 900$ (grey in colour) coating with the 'normal' finish and non-fouling control primer coating after one-month of exposure in the slime farm, respectively. Fouling developed on FRC replicates were light in coverage in comparison to the primer coatings. Additionally, small weed-like filaments were observed on the primer coatings.

The $2^{\text {nd }}$ campaign of slime growth tests on Intersleek ${ }^{\circledR} 900$ (red in colour) with mimicked 'hull' roughness densities started in March 2016 and was removed after 2 weeks of exposure time due to the restricted availability of the water tunnel used for the drag tests. Figure $4 \mathrm{~d}$ and $4 \mathrm{e}$ (red coatings) illustrate photos of biofilms collected from the $2^{\text {nd }}$ campaign of experiments in the slime farm on Intersleek ${ }^{\circledR} 900$ types with low and high hull roughness densities. The biofilms on these panels seemed to be more loosely attached and less homogeneous than those from the $1^{\text {st }}$ campaign, likely due to the limited exposure time.

Figure 4.Views of test panels before and after exposure in natural and laboratory habitats. Biofilm samples collected under field conditions before hydrodynamic tests (row a); The $1^{\text {st }}$ 
campaign of biofilm samples collected under laboratory conditions before hydrodynamic tests (rows b, c); The $2^{\text {nd }}$ campaign of biofilm samples collected under laboratory conditions before hydrodynamic tests (rows d, e).

\section{Biofilm parametrization}

Knowledge of the surface characteristics of the test panels covered with biofilms is essential for correlation with their drag performances. For this purpose, biofilm characterization expressed as biofilm \% surface area coverage and physical measures was carried out. An 'ImageJ' or Image Java processing and analysis program developed at the National Institutes of Health (Bethesda, Maryland, USA) was used to quantify the \% area coverage of biofilmed surfaces. Digital images of the biofilm-covered surfaces were taken using a Samsung Galaxy camera with model number: EK-GC100 with 16MP and optical zoom length of 21x. While taking images, a ruler was used as a scale bar. Known distances between the landmarks were later used to set a scale, so the area of selection was spatially calibrated in units of $\mathrm{mm}^{2}$. Once the images were loaded into ImageJ and the scale had been set, colour thresholding was applied to convert the images to grayscale images with user-defined brightness. After selection of contrasted biofilm covered particles, the total area was calculated. As an example, Figure 5 demonstrates the biofilm coverage area in percent for primer coating calculated using ImageJ software.

Figure 5. An image of 'Primer_Lab1' panel with 30\% of its area covered and highlighted in yellow with laboratory fouling samples.

Following the earlier outlined procedure, the area of fouling \% coverage for all panels covered with natural and laboratory biofilm samples is summarised in Table 1, which also gives an overall description of the fouling observed on each coated test panel. Physical roughness character, which is given in terms of peak-to-valley height or $R_{t}$ in $\mu \mathrm{m}$ on each biofilm covered surfaces was obtained using a laser-based Uniscan 100A Optical Surface Profilometer (Uniscan Instruments, Buxton, Derbyshire, UK) just before their installation in the water tunnel. Average $R_{t}$ values were calculated using 10 line profiles over biofilm covered areas with a $90 \mathrm{~mm}$ length and $25 \mu \mathrm{m}$ resolution for biofilm covered panels are presented in Table 1 . Average $R_{t}$ values include the combined $R_{t}$ value of the base coating roughness and biofilms. The total time required to conduct measurements for one panel was 10 to 15 minutes in order to avoid the dewatering and subsequent alteration of the biofilm structure. 
Table 1. Biofilm \% coverage area and mean peak-to-valley height $\left(R_{t}\right)$ for coated panels with representative biofilm samples

\begin{tabular}{|c|c|c|c|c|}
\hline & Test panels & $\begin{array}{l}\text { \% Total } \\
\text { biofilm } \\
\text { coverage }\end{array}$ & Fouling description & $\begin{array}{c}\text { Mean thickness } R_{t} \\
(\mu \mathrm{m})\end{array}$ \\
\hline \multicolumn{5}{|c|}{ Coated panels with natural biofilms } \\
\hline & I_900_Field1 & 27 & \multirow{2}{*}{ 6-month-old uneven, patchy and tenacious biofilm } & 443 \\
\hline & I_900_Field2 & 38 & & 300 \\
\hline \multicolumn{5}{|c|}{ Coated panels with laboratory biofilms } \\
\hline \multirow{4}{*}{ 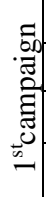 } & I_900_Lab1 & 4 & \multirow{2}{*}{ 1-month old fluffy biofilms } & 454 \\
\hline & I_900_Lab2 & 2 & & 550 \\
\hline & Primer_Lab1 & 30 & \multirow{2}{*}{$\begin{array}{l}\text { 1-month-old tough and moderate fouling with weed } \\
\text { like filaments }\end{array}$} & 1987 \\
\hline & Primer_Lab2 & 41 & & 2057 \\
\hline \multirow{4}{*}{ 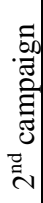 } & I_900_Lab_low1 & 42 & \multirow{4}{*}{ 2-week-old thin films of slime } & 472 \\
\hline & I_900_Lab_low2 & 12 & & 497 \\
\hline & I_900_Lab_high1 & 17 & & 417 \\
\hline & I_900_Lab_high2 & 5 & & 512 \\
\hline
\end{tabular}

\subsection{Flow measurement and estimation of wall shear stress}

In order to investigate the hydrodynamic drag characteristics of coated panels with fouling, boundary layer experiments were carried out in the Emerson Cavitation Tunnel (ECT) of Newcastle University. The velocity profiles in the boundary layer for flat panels were collected using a non-instrusive Laser Doppler Velocimetry (LDV). Velocity profile measurements were taken over 10 panels with biofouling and one reference acrylic smooth panel at position 1 (POS1), which corresponds to $2.7 \mathrm{~m}$ ( or $\approx 61 \delta$ ) from the leading edge of the tunnel's insert and $0.5 \mathrm{~m}$ from the test panel's leading edge as demonstrated in Figure 6. The location of POS1 ensures that there is some length for the flow to develop over the test panels. Velocity measurements were conducted in coincidence mode with minimum and maximum of 5000 and 20000 samples per measurement point, accordingly. The velocity-time data were collected for 60 seconds. Velocity components, which are in the streamwise, $U$ and wall-normal directions, $V$ were measured at varying distances from the surface (also called “wall”) and for the three incoming velocities. Each boundary layer profile consisted of 57 different vertical positions from the wall, the smallest step size between the two measuring positions was $25 \mu \mathrm{m}$. After insertion of each biofilm covered panel, but before LDV measurements were collected, the water in the tunnel was circulated at around $1 \mathrm{~m} / \mathrm{s}$ speed to remove loosely attached fouling. Details concerning the properties of optical FiberFlow LDV system can be found in Yeginbayeva I.A (2017).

Figure 6. 'I_900_Lab_low1' covered with 2-weeks old laboratory biofilms and FiberFlow LDV probe mounted on the traverse system before the BL measurements (a); The region of the superimposing laser beams or the measurement volume at POS1 (b). 
During data analysis, it was observed that the velocity profiles in the logarithmic region for some coated test panels with fouling eg heavy biofilms on the primer control coatings are distorted. Due to the effects of fouling on the shape of mean-velocity profiles, the use of velocity profile-fitting methods such as Krogstad et al. (1992) for the calculation of friction velocity $\left(u_{\tau}\right)$, hence the local skin friction coefficients $\left(C_{f}\right)$ for biofilm covered panels was found to be troublesome. Therefore, to avoid large uncertainties in the estimate, $u_{\tau}$ for the test panels covered with biofilms were obtained using the Reynolds shear stress analysis method. This method has been used in several studies on smooth and rough walls including Schultz (1998)and Lee, et al. (1993). In the present study, a plateau of constant Reynolds shear stress, $u^{\prime} v^{\prime}$ observed in the log-law region of velocity profiles was used for the analysis. Firstly, the normalised Reynolds shear stress given in Eq. (1) is plotted against a non-dimensionalised distance from the wall, $\frac{y}{\delta}$. In Eq. (1), $U_{e}$ is outer or freestream flow velocity, $u^{\prime}$ and $v^{\prime}$ represents the streamwise and wall normal velocity fluctuations of the measured boundary layer flow, respectively.

$$
\text { Reynolds shear stress }=\frac{-2\left(u^{\prime} v^{\prime}\right)}{U_{e}{ }^{2}} \quad \text { Eq. } 1
$$

Secondly, the skin friction coefficient, $C_{f}$, is estimated by drawing a trendline through the peak points of the Reynolds shear stress plot and getting the values at the intersection of this line with a vertical axis.

\subsection{Uncertainty analysis}

Precision based uncertainty estimates for the unfouled panel measurements were achieved through repeated tests based on the Coleman and Steele (1999). Six replicate LDV measurements were carried out for the rough FRC at POS1. For most of the boundary layer, $y / \delta>0.1$, the uncertainty in the streamwise velocity, $\mathrm{U}$, accounted for $\pm 1 \%$. The precision uncertainty in $u^{\prime} v^{\prime}$ was $\pm 4 \%$. The uncertainties in $C_{f}$, using the Reynolds stress method were calculated were $\pm 6.5 \%$. Drag measurements of fouled panels cannot be repeated as is done for newly applied or clean panels. Due to the constant change of surface characteristics, and thus drag, it is difficult to make a definitive statement on the uncertainty levels of the test with biofilm covered surfaces. It is believed that the uncertainty estimates associated with fouled panels are likely to increase due to flow instabilities triggered by the roughness changing with time.

\section{Results and Discussions}

\subsection{Boundary layer (BL) analysis}

In the experiments, a momentum thickness-based Reynolds number $(R e)$ ranges from $R e_{\theta}=7500$ to $R e_{\theta}=22000$ (smooth acrylic panel). During data analysis, the following boundary layer parameters were calculated for three streamwise velocities (nominally $2 \mathrm{~m} / \mathrm{s}, 4 \mathrm{~m} / \mathrm{s}$ and $6 \mathrm{~m} / \mathrm{s}$ ): the friction velocity, $u_{\tau}$ boundary layer thickness, $\delta$, displacement thickness $\delta^{*}$, momentum thickness, $\theta$, skin friction coefficient, $C_{f}$ and $R e$ number based on displacement thickness, $R e_{\delta^{*}}$, and momentum thickness, $R e_{\theta}$ for all test conditions. According to the complete data for the main flow and boundary layer flow parameters presented in Table 3 in the supplemental materials, the minimum and maximum displacement thickness values, $\delta^{*}$ for the panels covered with biofilms were 5.02mm (for I_900_Field1, 2m/s) and 6.76mm (for Primer_Lab1, 2m/s), respectively. High 
values in the displacement thickness $\left(\delta^{*}\right)$ as in the case of fouled primer coatings might be indicator of higher skin friction drag. Although the boundary layer thickness $(\delta)$ showed a higher degree of variability for biofilm covered panels, it appeared to be unaffected by the presence of heavy fouling on primer coated panels.

\subsection{Roughness functions}

Establishing the drag of any surface implies finding the velocity decrement caused by the surface roughness, which is known as the roughness function, $\Delta U^{+}$. The larger the $\Delta U^{+}$is, the more effect the roughness has on the mean profile and hence the wall shear stress. The $\Delta U^{+}$can be derived from the local skin friction coefficient, $C_{f}$, by comparing rough and smooth surfaces at the same $R e_{\theta}$ as shown in Eq. 2 (Granville 1987). The roughness function, which is a function of roughness Reynolds number $\left(k^{+}\right)$(or $\Delta U^{+}=f\left(k^{+}\right)$), is unique for every surface. Here $k^{+}=\frac{k u_{\tau}}{v}$. In the case of clean (or unexposed to fouling) surfaces, the roughness length scale, $k$, can be a single parameter or a combination of different roughness parameters representing the surface. In similar fashion, the biological roughness on fouled surfaces has to be described and taken into account. Once the roughness function for a given rough surface is known, it can be used to predict the drag of the ship with varying lengths using boundary layer similarity law method (Granville 1958, 1987).

$$
\Delta U^{+}=\left(\sqrt{\frac{2}{C_{f}}}-\sqrt{\frac{2}{C_{f m o o t h}}}{ }_{\text {rough }}\right)
$$

To form the basis for drag prediction in full-scale, analyzed $\Delta U^{+}$for test surfaces were derived from $C_{f}$ values obtained from the boundary layer data analysis (see the Section 2.5 ).

Based on the limited investigations and available data in the literature, the combination of the mean biofilm thickness and \% coverage is regarded as the most successful relationship in correlating the drag and physical nature of biofilm-covered surfaces. As an example, Schultz et al. (2015) used an effective roughness length, $k_{\text {eff }}$ based on the mean biofilm thickness taken as the roughness length scale $(k)$ and the biofilm \% coverage as presented in Eq.(3). The combination of these two parameters appeared to be effective in representing roughness functions for some but not all biofilm covered surfaces.

$$
k_{\text {eff }}=0.055 k(\% \text { coverage })^{\frac{1}{2}}
$$

Eq. 3

In this study authors adopted the approach by Schultz et al. (2015) and the roughness length scale ( $k$ ) of coatings with biofilms was represented by the mean biofilm thickness denoted by $R_{t}$ and total biofilm coverage (\% coverage). In Figure 7, one can see that the $\Delta U^{+}$of coated panels with biofilms for the present study are plotted as a function of roughness Reynolds number, $k^{+}$.

Figure 7. $\Delta U^{+}$plotted against $k^{+}$for various coated panels with naturally and laboratory-grown biofilms

Figure 7 also includes the roughness function of Lewkowicz and Das (1986) for model algae and two well-known roughness functions specifically developed for engineering surfaces: Colebrook 
(1939) and Grigson (1992) correlations. In a recent study Walker et al. (2013) investigated the structure of the turbulent boundary layer flows over biofouled test panels with smooth and rough substrata. They found that the roughness functions for fouled plate measurements in the hydraulically rough regime were in similar form to the logarithmic relationship of Lewkowicz and Das (1986). The roughness function of Walker et al. (2013) has also been included in Figure 7. Furthermore, Schultz (2007) assumed and validated that the roughness functions of a range of coating and fouling conditions showed the characteristic between the monotonic (Colebrook, 1939) and inflectional (Ligrani and Moffat 1986) roughness functions. This relationship, which was initially suggested for three-dimensional rough surfaces by Schultz and Flack (2007) is also incorporated in Figure 7 for comparison.

The general percentage formula was used to calculate the percent change in $C_{f}$ for fouled test panels in terms of the $C_{f}$ values for the smooth acrylic reference. As seen in Figure 7 the primer control coatings, which were exposed to fouling tests under laboratory conditions developed heavy filamentous fouling and displayed the highest increase in $C_{f}$, thus the highest value in $\Delta U^{+}$. It is shown in Figure 7 that the the first $\Delta U^{+}$data points for fouled primer control coatings (Primer_Lab1\& Primer_Lab2) fall between the Walker et al (2013) and the fully-rough asymptote roughness function lines. Whereas the slope for the rest $\Delta U^{+}$data points for Primer_Lab1\& Primer_Lab2 was negative. This downward shift in roughness function curve can be explained by the removal of fouling bulk fragments under high-flow conditions from primer coatings. The effect of the highest test velocity can be noticed from the reduction of the $C_{f}$ for Primer_Lab1 and Primer_Lab2 by $18 \%$ and $21 \%$ when compared to their initial fouling conditions, respectively. Figure 7 also reveals that after the water-jet cleaning of the remaining heavy filamentous slime from Primer_Lab1 and retesting, the $C_{f}$ for Primer 1 _cleaned decreased by $34 \%$ on average compared to its fouled condition, albeit slightly higher than the unfouled state. After the cleaning, most fouling was removed from the primer coating with the exception of remains especially in an array of roughness elements. This is supported by the transition of flow regimes from fully rough (in magenta circle) to transitionally-rough (in magenta star) for Primer 1_cleaned.

As far as the biofilms on Intersleek ${ }^{\circledR} 900 \mathrm{FRC}$ panels are concerned, in general it has been noticed that coatings with biofilm coverages starting from $10 \%$ to $40 \%$ display the inflectional roughness function behavior similar to the one proposed by Schultz and Flack (2007). These include natural biofilms with patchy coverage developed on I_900_Field2 and laboratory biofilms on mimicked 'low' and 'high' density of hull roughness (I_900_Lab_low2 and I_900_Lab_high1). Whereas, roughness functions for FRC panels with biofilms covering up to 5\% of panel areas are spread around the monotonic Colebrook-White curve proposed by Colebrook (1939). The rest of the tested data for surfaces I_900_Lab_low1 and I_900_Field1 with 37\% and 40\% of biofilm coverages respectively spread around curve of Ligrani and Moffat (1986). In summary, three distinct roughness function trends for biofilmed test panels were noticed depending on percent coverage and thickness of biofilms on FRCs.

\subsection{Effects of coating roughness and biofilms on ship performances}

Schultz (2007) presented a simple and effective algorithm of Granville's methodology to predict the effect of different ranges of coating roughness and biofouling on the resistance of a full-scale ship. By following this algorithm, the effect of given roughness on the frictional drag of plates of 
two ship lengths were predicted in order to model the change in a coefficient of frictional drag, $C_{F}$, and a power, $\Delta P_{E}$ of full-scale ships. Assumptions are being made, such as that the ship hulls are covered with the same roughness observed on test panels in laboratory scale (ie combined effects of Intersleek® 900 FRC roughness and biological roughness) and the pressure drag of selected ships are not greatly affected by the roughness.

The first ship selected is a KRISO Container Ship (KCS) with a waterline length of 232.5m (Kim et al. 2001). Recent paper by Yeginbayeva I A and Atlar (2018) predicted the effect of marine coatings with so-called 'normal' and mimicked 'hull' application finishes on the frictional resistance and effective power of this particular ship using the classical procedure of Granville. This work also presented new roughness allowances associated with mentioned coating finishes. The current paper builds on prior study by generating frictional resistance and effective power data for the KCS with 'in-service' hull conditions. In addition, the change in the frictional resistance and power penalties due to the presence of fouling were also estimated for a bulk carrier with a length of 175m ('Bulk carrier 1'). Smooth hull data for this ship were kindly provided by Dr. Demirel (personal communication, February 2017).

\section{KRISO Container Ship (KCS) and Bulk Carrier performance data}

The drag associated with roughness, either physical or biological, decreases the momentum of the flow. This loss of momentum is described by the $\Delta U^{+}$, which in this study represents the combined effects of a coating's inherent roughness and biological roughness on the mean velocity profiles obtained from the detailed boundary layer measurements with the panels in the re-circulating water channel. Granville's similarity law procedure (Granville 1987) was used to scale up the $\Delta U^{+}$to predict a coefficient of frictional drag, $C_{F}$, for the selected full scale ships. Initially, the smooth friction line, $C_{\text {Fsmooth }}$, is calculated using the formula of Schlichting (1968) in logarithmic structure for each ship (flat-plate approach with the same length of a ship) to be plotted as a function of Reynolds number $\log \left(R e_{L}\right)$. The frictional drag coefficient for the rough surface, $C_{\text {Frough }}$, is determined by displacing the $C_{F s m o o t h}$ in the positive $\log \left(R e_{L}\right)$ direction by a distance of $\Delta U^{+} \mathrm{K}\lfloor\ln (10)]^{-1}$. Here, $\mathrm{K}$ is the von Karman constant, which is equal to 0.41. Having calculated the desired ship length scales, $L^{+}$ship scale, the scaled up results for a full-scale ship can be read from the intersection of the $L^{+}$ship scale line and the $C_{\text {Frough }}$ line. The added drag, $\Delta C_{F}(\%)$, due to the presence of the roughness can be presented as in Eq.(5). $\Delta C_{F}$ is also known as a 'roughness allowance' in the traditional power prediction of ships and attributed to the real life, full-scale hull surface conditions.

$$
\Delta C_{F}(\%)=\frac{C_{\text {Frough }}-C_{\text {Fsmooth }}}{C_{\text {Fsmooth }}} \times 100
$$

Eq. 4

The results of the extrapolation study presented in Figure 8 and Table 2 indicate that the effects of biofilms, predicted based on the laboratory- or naturally-grown approach, are significant. From Figure 8, it can be seen that the added drag coefficients in percent, $\left(\% \Delta C_{F}\right)$, due to the presence of biofilms on the KCS hull having similar drag characteristics to the Intersleek ${ }^{\circledR} 900$ FRC-coated panels varied between $9.5 \%$ and $20 \%$ depending on biofilm coverages. When extrapolated to the full-scale KCS hull, 6-month-old tenacious field-grown biofilm samples with 27\% cover (I_900_Field1) and 37\% cover (I_900_Field2) caused increases of $\Delta C_{F}$ ranging from $13 \%$ to $19 \%$ when compared to the smooth hull data. Similarly, the fluffy biofilms grown under laboratory 
conditions resulted in 12\% (I_900_Lab2) and 14\% (I_900_Lab1) increase in $\Delta C_{F}$ for the KCS. As demonstrated by Yeginbayeva I A and Atlar (2018), a small increase, on average $2 \%$ and $3 \%$ in $\Delta C_{F}$ is expected from the smooth FRC on the KCS at 19 knots and 24 knots, respectively. So the presence of laboratory biofilms the FRC-coated panels with 'normal' finish caused from four to seven-fold greater added drag $\left(\Delta C_{F}\right)$ than the FRC panels with 'normal' finish and fouling-free condition. As shown in Figure 8a and Table 2, the minimum and maximum increase in $\Delta C_{F}$ for FRC replicates due to the combined effects of 'low/micro' roughness density and 2-weeks old biofilms were $13 \%$ and $20 \%$, which is equivalent to 1.9 and 2.9 times higher drag as compared to their clean (no biofilm) conditions. In the scenario, when the KCS hull is covered by the fluffy biofilms with underlying 'high/macro' FRCs roughness densities, predicted results indicate $10 \%$ and 19\% drag increase $\left(\Delta C_{F}\right)$ for I_900_Lab_high1 and I_900_Lab_high2, respectively.

According to the results presented in Table 2, large differences in the drag performance were observed for the condition when the hull is covered by heavy weed fouling between two different KCS's speeds (i.e 19 knots with 24 knots). As expected, the added drag for fouled primer coatings was significantly higher than for fouled FRCs with different roughness densities. The increase in $\Delta C_{F}$ for the primer coatings with heavy fouling dominated mainly by weed filaments was about 57\% (Primer_Lab1) and 62\% (Primer_Lab2) at 19 knots, which changed to 56\% (Primer_Lab1) and 71\% (Primer_Lab2) at 24 knots.

In general, the drag penalties for the primer coatings with heavy fouling observed with bulk carrier at 12 knots are slightly higher than those predicted for the KCS hull with the same coating conditions at 19 knots and 24 knots. In this comparison, apart from the slower design speed of the bulk carrier, its shorter waterline length (i.e. $175 \mathrm{~m})$ than that of KCS $(232.5 \mathrm{~m})$ is to be born in mind.

Figure 8. Estimation of percent change in frictional resistance or added drag $\left(\% \Delta C_{F}\right)$ for the KCS and bulk carrier at investigated speeds with the presence of biofilms on Intersleek ${ }^{\circledR} 900$ type FRCs with different roughness densities 
Table 2. Change in added drag $\left(\% \Delta C_{F}\right)$ or roughness allowance $\left(\Delta C_{F}\right)$ and effective power $\left(\% \Delta P_{E}\right.$ ) for the KCS and bulk carrier due to the presence of biofilms on Intersleek ${ }^{\circledR} 900$ type FRCs with different roughness densities

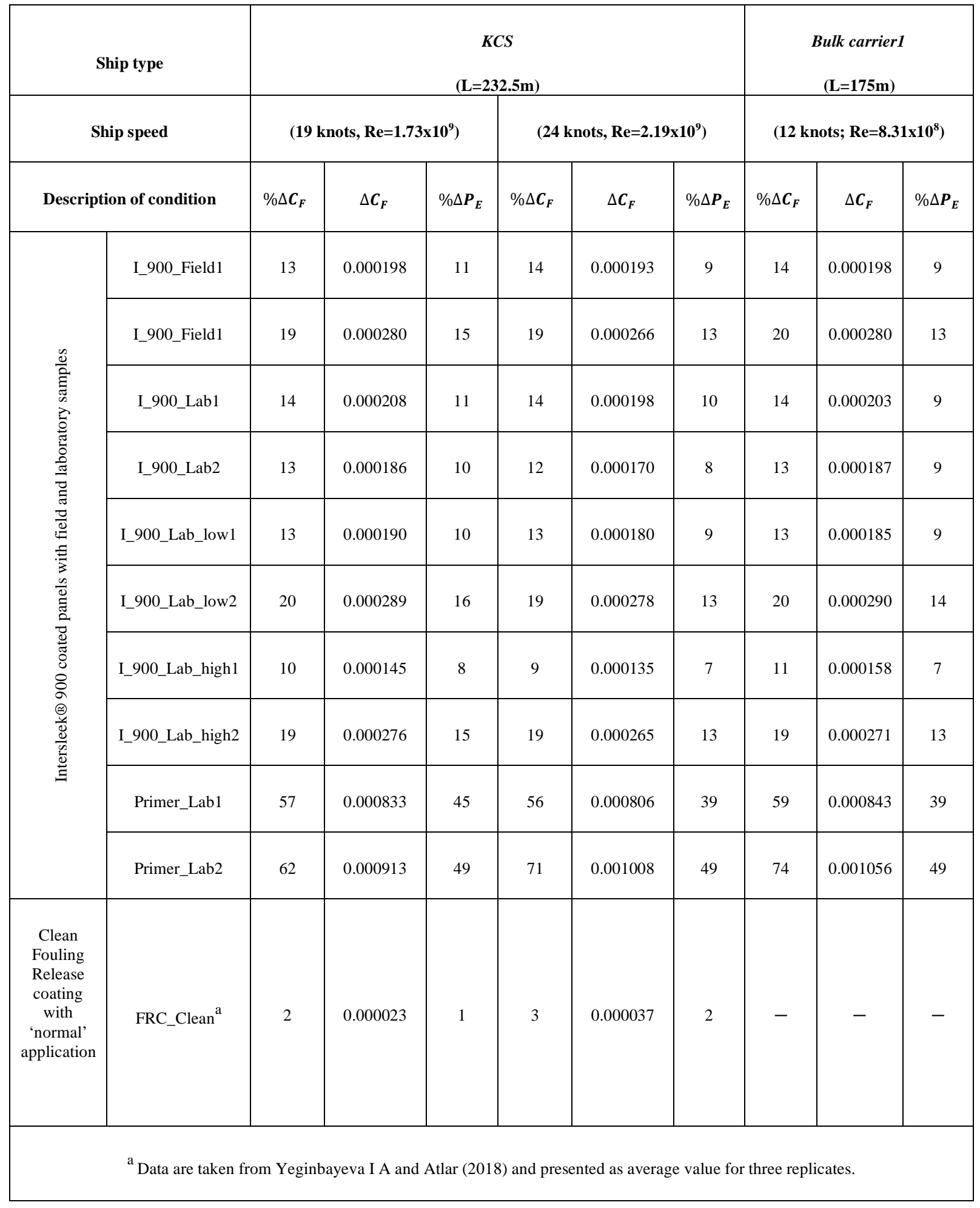




\section{Conclusions}

The present study investigated an important part of 'in-service' hull conditions such as coating roughness and biofilms, which in combination contribute to drag increase. The aim of the study was to investigate the combined effect of roughness and biofilms on the frictional resistance and powering characteristics of a typical container and bulk carrier in full-scale. In achieving the above aim, the similarity law scaling procedure of Granville was utilized to extrapolate the skin friction values (i.e. roughness functions) obtained from experimental boundary layer data to full-scale ship values of Reynolds number. Based on the analysis of results, the following summary of findings are obtained:

1. Two alternative means of biofilm cultivation on flat test panels were explored by a purposebuilt device. The first approach is based on the deployment of coated panels from the strut assembly of the UNEW research vessel and gives an advantage of developing natural biofilm samples under real sea exposure conditions. Whereas the second approach is based on developing representative biofilms in a more practical manner in a slime cultivating farm during shorter periods and under a controlled environment. Both devices were capable of simulating the first stages of biofouling (or biofilms) on FRC-coated panels to predict the effects of 'in-service' coating conditions. No direct comparisons can be made between the field and laboratory-based biofilms since no correlation factor is available which accounts for and relates the growth rates for the short interval and longer exposure periods;

2. Substantial differences in the structure of the biofilms were observed between slime samples collected at sea and grown in controlled laboratory conditions. Biofilms collected in the laboratory using the slime farm were fluffy in structure and easily removed at the highest shear flow in the water tunnel. On the contrary, the natural biofilm samples collected at open sea were tenacious. It appears that organisms attached to FRC coated panels turn on stressresponse genes and render the biofilms more resistant when exposed to environmental stresses. This makes biofilms on FRC coated panels exposed at sea different in sloughing compared to the biofilms on FRC coated panels exposed in the laboratory. Photos taken after the hydrodynamic tests (see supplemental Figure 9) demonstrates that the sloughing was immediately apparent for FRC coated panels with laboratory-grown biofilms. As far as the biofilm characterization is concerned, the mean thickness of biofilms was measured only before the drag measurements. The constant changes in biofilm coverage and mean thickness pose difficulties in interpreting its effect on ship powering penalties with confidence. Therefore, the presented extrapolated results to the ship scale Reynolds number values (in Table 2) must be approached with some caution;

3. Substantial differences in appearance and distribution of the fouling, as well as drag performance, were observed between the non-fouling primer coated panels and FRC-coated panels exposed to fouling growth in the same slime farm. The ' 1 st campaign' of laboratory experiments revealed that FRC-coated panels are extremely effective against settlements of heavy weed-like filament in contrast with primer coated control panels. The significant 
increase in $\Delta C_{F}$ (45\% 74\%) can be attributed to the tenacious weed-like filaments developed only on the primer coated panels. The percentage effective power penalty ( $\% \Delta P_{E}$ ) due to the same weed-like fouling ranged from around $39-49 \%$. As discussed in Murphy et al. (2018), three dimensional flapping of filaments may produce larger effective roughness height than the physical roughness thus resulting in higher drag penalty. Schultz (2007), in his predictions of full-scale resistance and powering for mid-sized naval surface combatant, indicated that the 'small calcareous fouling or weed' can lead up to 34\% increase in shaft power at combatant cruising speed of 15 knots. Although the direct comparisons between these results and present results are impractical due to different ship lengths, fouling coverages and ship speeds, still, studies agree that the weed fouling exerts a detrimental effect on ship performance.

4. As far as the drag performances of the 'normal' finish FRCs with biofilms is concerned, there is a clear increase in $\% \Delta C_{F}$ and $\% \Delta P_{E}$ due to the presence of biofilms. The laboratory grown fluffy biofilms with little visible biofilm coverage (2\% to $4 \%$ ) are predicted to cause from $\sim 12 \%$ to $\sim 14 \%$ of added drag, $\% \Delta C_{F}$. In comparison, tenacious, sticky biofilm samples grown at sea during 6-months (27\% to $38 \%$ area coverage) caused from $13 \%$ to $20 \%$ increase in $\% \Delta C_{F}$.Given the relatively short timeframe and relatively slower speeds, under which the laboratory biofilm samples were developed as opposed to natural biofilms, these penalties are significant and are believed to result mainly from patchy but thick biofilms. The biofilms grown on coated panels deployed from the strut assembly of the vessel can be regarded as good representatives of real-world biofilms, since their deployment was according to the real sea conditions in terms of the depth of submergence, the availability of nutrients, salinity and sea temperature. In general, the increase in $\% \Delta C_{F}$ calculated for KCS hull at 19 knots due to the combined effects of 'normal' finish coating roughness and biofilms is 7-8 times higher than the predicted values for the same coating finish on KCS hull but in clean condtions (refer to the footnote in Table 2);

5. The drag effects of combined effects of 'hull' roughness and laboratory- grown biofilms were explored for the first time. The findings indicate that the drag penalties resulting from this combined effect of biofilms and mimicked 'hull' roughness ranges are greater than the isolated case with no biofilms. Based on the two case study findings, roughness allowance $\left(\Delta C_{F}\right)$ ranges for two ship types which have an example of a commercial FRC with more representative of hull roughness ranges on their surface are suggested. This updated $\Delta C_{F}$ may have the potential to be used as a correction factor to account for the hull roughness effect.

5.1. $0.00014<\Delta C_{F}<0.00029$ for the typical container ship with FRC with 'micro/macro' hull roughness and fluffy biofilms;

5.2. $0.00016<\Delta C_{F}<0.00029$ for the typical bulk carrier with FRC with 'micro/macro' hull roughness and fluffy biofilms; 
These above stated roughness allowance additive to the full-scale value of $C_{F}$, can be attributed to surface roughness of typical hulls due to fluffy biofilms developed at flow velocities $<1 \mathrm{~m} / \mathrm{s}$ with the fouling rating from 10 (i.e incipient slime) to 20 (advanced slime) according to the fouling classification scale provided by the U.S. Navy (2006). Whereas, the roughness allowance $\left(\Delta C_{F}\right)$ ranges presented below can be added to the full-scale value of $C_{F}$ for typical ship types to account for more resilent and representative biofilms on FRCs with fouling rating scale of 10 to 20:

5.3. $0.00019<\Delta C_{F}<0.00028$ for the typical container ship with FRC with 'normal' hull roughness and tenecious biofilms;

5.4.0.0002 $<\Delta C_{F}<0.00028$ for the typical bulk carrier with FRC with 'normal' hull roughness and tenecious biofilms;

6. The data presented can also be used as modern benchmark data to support and further enhance the CFD approaches in terms of the wall functions used (e.g. Demirel et al. (2017)) to predict the effect of FRC 'in-service' conditions. The findings of the study, therefore, are an important addition to the body of knowledge on the 'in-service' performance of modern hull coatings, in particular, for the FRC, in terms of providing more realistic data samples and using these data to demonstrate its effect on ship hull performance. However, with a small number of replicates, caution must be applied, as the findings are valid for the surface treatments and exposure conditions in which the experiment was done and might not be transferable to all cases. In terms of future work, it would be interesting to carry out single experiments with many replications or several repeated experiments with fewer replications. The strength of comparison across treatments are limited by the colour differences between Intersleek ${ }^{\circledR} 900$ grey panels and Intersleek ${ }^{\circledR} 900$ red panels. The study carried out by Dobretsov et al. (2013) investigated the effect of the substratum color (white and black) on the formation of marine micro and macrofouling communities in tropical waters. The study showed that early settlement on white and black slides was uneven, but eventually the microfouling load evened out across coating colors (black and white). However, the impact of substratum colour on the formation of biofouling communities diminished over time (several months exposure). Therefore, based on the study of Dobretsov et al. (2013), the authors expect biofouling communities on Intersleek ${ }^{\circledR} 900$ grey panels (lighter color) exposed up to 6 months in the North Sea to be roughly equivalent to Intersleek ${ }^{\circledR} 900$ red panels.

\section{Acknowledgments}

Authors gratefully acknowledge the funding received from International Paint Ltd, a division of AkzoNobel. Special thanks go to Dr David Williams, Dr Barry Kidd, Dr Philip Stenson for their financial support, valuable input and supervisory role. Special thanks also go to Peter Hobson from HobsonDesign for taking the slime farm concept from the drawing board to its successful completion, to Dr Jeremy Thomason for his insightful comments and advice which were of immense help in the cultivation of biofilm samples.

\section{Funding}

This research was funded by International Paint Ltd (a division of AkzoNobel) and Newcastle University. 


\section{References}

Anderson C, Atlar M, Callow M, Candries M, Townsin RL. 2003. The Development of FoulRelease Coatings for Seagoing Vessels. Journal of Marine Design and Operations. No. B4:11-23.

Andrewartha J, Perkins K, Sargison J, Osborn J, Walker G, Henderson A, Hallegraeff G. 2010. Drag Force and Surface Roughness Measurements On Freshwater Biofouled Surfaces [Research Support, Non-U.S. Gov't]. Biofouling. 26(4):487-496. eng.

ARBO. 2016. ARBO pompen en filters BV. Leemdijk 2, 9422 CL SMILDE - The Netherlands; [accessed].info@arbo-pumps.com.

Atlar M, Bashir M, Turkmen S, Yeginbayeva I, Carchen A, Politis G. 2015. Design, Manufacture and Operation of a Strut System Deployed on a Research Catamaran to Collect Samples of Dynamically Grown Biofilms In-Service. Proceedings of the 4th International Conference on Advanced Model Measurement Technology for Maritime Industry (AMT'15); Istanbul, Turkey.

Benson JM, Ebert JW, Beery TD. 1938. Investigation In The NACA Tank Of The Effect Of Immersion In Salt Water On The Resistance Of Plates Coated With Different Shipbottom Paints. Washington (DC): National Advisory Commitee on Aeronautics.

Characklis WG. 1973. Attached Microbial Growths-1. Attachment and Growth. Water Research. 7(8):1113-1127.

Colebrook CF. 1939. Turbulent Flows in Pipes, with Particular Reference to Transition Region between Smooth and Rough Pipe Walls. Journal of Civil Engineers. 11:133-157.

Coleman HW, Steele WG. 1999. Experimentation and Uncertainty Analysis for Engineers. Third ed. John Wiley \& Sons. Inc.

Demirel YK, Turan O, Incecik A. 2017. Predicting the Effect of Biofouling on Ship Resistance using CFD. Applied Ocean Research. 62(2017):100-118.

Denny ME. 1951. B.S.R.A resistance experiments on the Lucy Ashton: part 1- full-scale measurements. Trans INA. 93:40-57.

Dobretsov S, Abed RMM, Voolstra CR. 2013. The effect of surface colour on the formation of marine micro and macrofouling communities. Biofouling. 29(6):617-627.

Granville PS. 1958. The Frictional Resistance and Turbulent Boundary Layer of Rough Surfaces. Journal of Ship Research. 2:52-74.

Granville PS. 1987. Three Indirect Methods for the Drag Characterization of Arbitrarily Rough Surfaces on Flat Plates. Journal of Ship Research. 31:70-77.

Grigson CWB. 1992. Drag Losses of New Ships Caused by Hull Finish. Journal of Ship Research. 36(2):182-196. 
Haslbeck EG, Bohlander GS. 1992. Microbial Biofilm Effects on Drag-Lab and Field. Proc SNAME Ship Production Symposium; New Orleans, Louisiana.

Holland R, Dugdale TM, Wetherbee R, Brennan AB, Finlay JA, Callow JA, Maureen EC. 2004. Adhesion and Motility of Fouling Diatoms on a Silicone Elastomer. Biofouling. 20(6):323-329.

Kim WJ, Van SH, Kim DH. 2001. Measurement of Flows around Modern Commercial Ship Models. Experiments in Fluids. 31(5):567-578.

Krogstad PA, Antonia RA, Browne LWB. 1992. Comparison Between Rough and Smooth-Wall Turbulent Boundary Layers. Journal of Fluid Mechanics. 245:599-617.

Lewkowicz AK, Das DK. 1986. Turbulent Boundary Layers on Rough Surfaces With and Without a Pliable Overlayer: a Simulation of Marine Fouling. International Shipbuilding Progress. 33(386):174-186.

Lewthwaite JC, Molland AF, Thomas KW. 1985. An Investigation Into the Variation of Ship Skin Frictional Resistance with Fouling. Transactions of Royal Institute of Naval Architects. 127:269284.

Ligrani PM, Moffat RJ. 1986. Structure of transitionally rough and fully rough turbulent boundary layers. Journal of Fluid Mechanics. 162:69-98.

Lindholdt A, Dam-Johansen K, Yebra D, Olsen SM, Kiil S. 2015. Estimation Of Long-Term Drag Performance of Fouling Control Coatings Using Ocean-Placed Raft with Multiple Dynamic Rotors. JCoatTechnolRes. 12(6):975-995.

McEntee W. 1915. Variation of Frictional Resistance of Ships with Condition of Wetted Surface. Trans SNAME. 24:37-42.

Murphy EAK, Barros JM, Schultz MP, Flack KA, Steppe CN, Reidenbach MA. 2018. Roughness effects of diatomaceous slime fouling on turbulent boundary layer hydrodynamics. Biofouling. 34(9):976-988.

Navy US. 2006. Naval Ships' Technical Report: Waterbourne Underwater Hull Cleaning of Navy Ships.

Rodney MD. 2002. Biofilms: Microbial Life on Surfaces. Emerging Infectious Deseases. 8(9):881890.

Schlichting H. 1968. Boundary Layer Theory. New York: McGraw-Hill.

Schultz MP. 1998. The Effect of Biofilms on Turbulent Boundary Layer Structure $[\mathrm{PhD}$ Dissertation]. Florida Institute of Technology.

Schultz MP. 2004. Frictional Resistance of Antifouling Coating Systems. Journal of Fluids Engineering. 126:1039-1047. 
Schultz MP. 2007. Effects Of Coating Roughness And Biofouling On Ship Resistance and Powering. Biofouling. 23(5):331-341. eng.

Schultz MP, Bendick JA, Holm ER, Hertel WM. 2011. Economic Impact of Biofouling On A Naval Surface Ship. Biofouling. 27(1):87-98.

Schultz MP, Flack KA. 2007. The Rough-Wall Turbulent Boundary Layer from the Hydraulically Smooth to the Fully Rough Regime. Journal of Fluid Mechanics. 580:381-405.

Schultz MP, Swain GW. 2000. The Influence of Biofilm on Skin Friction Drag. Biofouling. Vol 15(1-3:129-139.

Schultz MP, Walker JM, Steppe CN, Flack KA. 2015. Impact Of Diatomaceous Biofilms On The Frictional Drag Of Fouling-Release Coatings. Biofouling. 31(9-10):759-773.

Townsin RL. 2003. The Ship Hull Fouling Penalty. Biofouling. 19 Suppl:9-15. eng.

Walker JM, Sargison JE, Henderson AD. 2013. Turbulent Boundary-Layer Structure of Flows Over Freshwater Biofilms. Exp Fluids. 54(1628):1-17.

Watanabe S, Nagamatsu N, Yokoo K, Kawakami Y. 1969. The Augmentation in Frictional Resistance due to Slime. J Kansai Soc Naval Architects. 131:45-51.

Yebra DM, Kiil S, Dam-Johansen K. 2004. Antifouling technology-past, present and future steps towards efficient and environmentally friendly antifouling coatings [Review]. Progress in Organic Coatings. 50(2):75-104.

Yeginbayeva IA. 2017. An Investigation Into Hydrodynamic Performance of Marine Coatings 'InService' Conditions [PhD thesis]. Newcastle, UK: Newcastle University.

Yeginbayeva IA, Atlar M. 2018. An experimental investigation into the surface and hydrodynamic characteristics of marine coatings with mimicked hull roughness ranges. Biofouling. 34(9):10011019.

Zargiel KA, Swain GW. 2014. Static VS Dynamic Settlement And Adhesion Of Diatoms To Ship Hull Coatings. Biofouling. 30(1):115-129. 